\title{
The role of the Grb2-p38 MAPK signaling pathway in cardiac hypertrophy and fibrosis
}

\author{
Shaosong Zhang, ${ }^{1,2}$ Carla Weinheimer, ${ }^{1,2}$ Michael Courtois, ${ }^{1,2}$ Attila Kovacs, ${ }^{1,2}$ \\ Cindy E. Zhang,,$^{1,2}$ Alec M. Cheng, ${ }^{2,3}$ Yibin Wang, ${ }^{4}$ and Anthony J. Muslin ${ }^{1,2,5}$ \\ ${ }^{1}$ Center for Cardiovascular Research, \\ ${ }^{2}$ Department of Medicine, and \\ ${ }^{3}$ Department of Pathology, Washington University School of Medicine, St. Louis, Missouri, USA \\ ${ }^{4}$ Department of Pharmacology, University of Maryland School of Medicine, Baltimore, Maryland, USA \\ ${ }^{5}$ Department of Cell Biology \& Physiology, Washington University School of Medicine, St. Louis, Missouri, USA
}

\begin{abstract}
Cardiac hypertrophy is a common response to pressure overload and is associated with increased mortality. Mechanical stress in the heart can result in the integrin-mediated activation of focal adhesion kinase and the subsequent recruitment of the Grb2 adapter molecule. Grb2, in turn, can activate MAPK cascades via an interaction with the Ras guanine nucleotide exchange factor SOS and with other signaling intermediates. We analyzed the role of the Grb2 adapter protein and p38 MAPK in cardiac hypertrophy. Mice with haploinsufficiency of the Grb2 gene (Grb2 ${ }^{+/-}$mice) appear normal at birth but have defective $T$ cell signaling. In response to pressure overload, cardiac $\mathrm{p} 38$ MAPK and JNK activation was inhibited and cardiac hypertrophy and fibrosis was blocked in $\mathrm{Grb2}^{+/-}$mice. Next, transgenic mice with cardiac-specific expression of dominant negative forms of $\mathrm{p} 38 \alpha(D N-p 38 \alpha)$ and $\mathrm{p} 38 \beta(\mathrm{DN}-\mathrm{p} 38 \beta)$ MAPK were examined. DN-p38 $\alpha$ and DN-p38 $\beta$ mice developed cardiac hypertrophy but were resistant to cardiac fibrosis in response to pressure overload. These results establish that Grb2 action is essential for cardiac hypertrophy and fibrosis in response to pressure overload, and that different signaling pathways downstream of Grb2 regulate fibrosis, fetal gene induction, and cardiomyocyte growth.
\end{abstract}

J. Clin. Invest. 111:833-841 (2003). doi:10.1172/JCI200316290.

\section{Introduction}

Human cardiac hypertrophy is a common condition that often develops as a by-product of hypertension or valvular heart disease. Adult cardiomyocytes are unable to divide, but respond to stress and growth stimuli by enlarging (1). Cardiac hypertrophy is associated with an increased risk of cardiac arrhythmias, diastolic dysfunction, congestive heart failure, and death $(2,3)$.

Pressure overload activates a wide variety of signaling pathways in cardiac tissue. For example, increased wall stress leads to the local release of ligands such as angiotensin II and endothelin-1 $(1,4)$. The precise requirement for these ligands in the hypertrophic

Received for publication June 26, 2002, and accepted in revised form December 17, 2002.

Address correspondence to: Anthony J. Muslin, Washington University School of Medicine, 660 South Euclid Avenue, Campus Box 8086, St. Louis, Missouri 63110, USA. Phone: (314) 747-3525; Fax: (314) 362-0186;

E-mail: amuslin@im.wustl.edu.

Conflict of interest: The authors have declared that no conflict of interest exists.

Nonstandard abbreviations used: focal adhesion kinase (FAK); extracellular signal-regulated kinase 1/2 (ERK); atrial natriuretic factor (ANF); Src-homology type 3 (SH3); $\alpha$-myosin heavy chain $(\alpha$-MHC); transverse aortic constriction (TAC); left ventricular (LV); sarcoplasmic-endoplasmic reticulum calcium ATPase (SERCA); left ventricular weight to body weight ratio (LVW/BW); left ventricular mass to body weight ratio (LVM/BW); MAPK kinase kinase (MAPKKK); Grb2-associated binder 1 (Gab1). process is not completely understood at this time, although angiotensin II receptor blockade inhibits the development of aortic banding-induced murine cardiac hypertrophy in a fashion that is independent of its ability to lower blood pressure (5).

In addition to causing the local release of ligands, pressure overload may also activate intrinsic "stretch" receptors in cardiomyocytes. Leading candidates for cardiac stretch receptors include the integrin family of transmembrane proteins (6-8). Outside-in integrin signaling is mediated by the binding of extracellular matrix proteins to an $\alpha \beta$ heterodimeric integrin pair (9-12). Binding of a matrix protein to an integrin heterodimer typically results in the activation of the nonreceptor tyrosine kinase, focal adhesion kinase (FAK) (6). Activated FAK, in turn, recruits the non-receptor tyrosine kinase $\mathrm{c}-\mathrm{Src}$, the multifunctional adapter molecule Grb2, and other signaling intermediates $(6,8,13)$.

Recent studies demonstrated that integrin activation, in concert with $G$ protein activation, might be essential for the growth of cardiomyocytes. For example, balloon-mediated ventricular stretch of isolated adult rat heart results in the rapid activation of FAK, promotes the association of Grb2 with FAK, and causes the activation of extracellular signal-regulated kinase $1 / 2$ (ERK) (14). In addition, stretch of cultured rat neonatal cardiomyocytes results in the rapid activation of FAK and c-Src, the formation of a Grb2-FAK complex, and the activation of p38 MAPK (8). Furthermore, 
expression of an integrin $\beta_{1 \mathrm{D}}$ inhibitor in cultured rat neonatal cardiomyocytes blocks phenylephrineinduced hypertrophy and atrial natriuretic factor (ANF) expression (7). Finally, expression of a dominant negative form of FAK, or expression of a mutant form of FAK that is unable to bind to Grb2, in cultured cardiomyocytes blocks phenylephrine-induced hypertrophy and ERK activation $(6,7)$.

Cardiac pressure overload results in the activation of integrin/FAK-mediated responses, but the importance of downstream components of this signaling pathway in the hypertrophic process remains to be determined. Recently, mice were developed with targeted disruption of the Grb2 gene (15). Grb2 is a scaffolding protein that contains two Src-homology type 3 (SH3) domains that flank a single SH2 domain $(16,17)$. SH3 domains bind to polyproline peptide motifs, and $\mathrm{SH} 2$ domains bind to phosphotyrosine-containing peptide motifs $(18,19)$. The SH3 domain of Grb2 binds to a polyproline motif on the Ras guanine nucleotide exchange factor SOS, and the SH2 domain of Grb2 binds to phosphotyrosine motifs present on activated FAK, Shc, and receptor tyrosine kinases. Grb2 $2^{-/-}$mice do not survive embryonic development due to defective endoderm differentiation and because they are unable to form the epiblast. However, $\mathrm{Grb2}^{+/-}$mice survive embryogenesis, appear normal at birth, and are fertile. Grb2 $2^{+-}$mice have a $40-50 \%$ reduction in Grb2 protein in all tissues tested to date and have a defect in $\mathrm{T}$ cell signaling (20). Although ERK activation is normal in $\mathrm{Grb2}^{+/-} \mathrm{T}$ cells, p38 MAPK and JNK activation is markedly reduced. Based on these defects in signal transduction, we thought $\mathrm{Grb2}^{+/-}$mice would be an excellent model system in which to study cardiac hypertrophy.

\section{Methods}

$\mathrm{Grb2}^{+/-}$mice. Grb2 $2^{+/-}$mice in the $129 / \mathrm{SvJ}$ strain were generated as previously described (15). Grb2-/- mice do not survive embryonic development. Grb2 ${ }^{+/-}$mice were compared with Grb2+/+ $129 / \mathrm{Sv}$ littermates in all experiments described here.

All research involving the use of mice was performed in strict accordance with protocols approved by the Animal Studies Committee of Washington University School of Medicine.

Transgenic mice with cardiac-specific expression of dominant negative forms of $p 38 \alpha$ or $p 38 \beta M A P K$. The coding region of the cDNA's of human DN-p38 $\alpha$ MAPK or DN-p38 $\beta$ MAPK $(21,22)$ were subcloned into a vector containing the $\alpha$-myosin heavy chain $(\alpha-\mathrm{MHC})$ promoter and an SV40 polyadenylation site as previously described (23). Linearized DNA was injected into the pronuclei of onecell Black Swiss embryos. Progeny were analyzed by PCR to detect transgene integration (24). Multiple lines in the Black Swiss genetic background were obtained for the construct, and integration of the transgene was analyzed by dot blot analysis. The highest-expressing lines, with integration of ten copies of the DN-p38 $\alpha$ transgene and 15 copies of the DN-p38 $\beta$ transgene, were used in this study. Transgenic DN-p38 $\alpha$ or DN-p38 $\beta$ mice were compared with congenic nontransgenic Black Swiss mice in every experiment.

Transverse aortic constriction. Transverse aortic constriction (TAC) was performed as previously described $(25,26)$. The surgeon was blinded in all cases to the transgenic status of the mice. After 7 days, surviving animals were subjected to transthoracic echocardiography and cardiac catheterization to determine cardiac function and proximal aortic pressure. Animals were then killed and the hearts were dissected out and weighed (24).

Transthoracic echocardiography. Transthoracic echocardiography was performed in awake mice by use of an Acuson Sequoia 256 Echocardiography System equipped with a $15-\mathrm{MHz}$ transducer (model 15L8) as described previously (Siemens Medical Solutions, Mountain View, California, USA) $(24,27)$. The echocardiographer was blinded in all cases to the transgenic status of the mice.

Cardiac catheterization. Closed-chest cardiac catheterization was performed as previously described (24). Continuous aortic pressure and left ventricular (LV) systolic and diastolic pressures were recorded on a Gould chart recorder (Gould Instruments, Valley View, Ohio, USA).

Histological analysis. Seven days after TAC, $\mathrm{Grb2}^{+/-}$and Grb2 $2^{+++} 129 /$ SvJ, DN-p38 $\alpha$, DN-p38 $\beta$, and nontransgenic Swiss Black mice were sacrificed and LV tissue was obtained. Tissue was fixed in 10\% formalin, embedded in paraffin, and sectioned with a microtome. Tissue sections were stained with $\mathrm{H} \& \mathrm{E}$ or with Masson trichrome (24).

Quantitative real-time RT-PCR. RNA was purified from quick-frozen cardiac tissue by use of the RNeasy protocol (QIAGEN Inc., Valencia, California, USA). The TaqMan Gold RT-PCR kit was used according to the manufacturer's instructions (Applied Biosystems, Foster City, California, USA). Quantitative PCR was performed by use of real-time detection technology and analyzed on a model 7700 Sequence Detector (Applied Biosystems) with specific primers and fluorescent probes for sarcoplasmic-endoplasmic reticulum calcium ATPase (SERCA), medium chain acyl-CoA dehydrogenase, ANF, and $\beta$-myosin heavy chain ( $\beta$-MHC). Levels of mRNA were compared at various timepoints after correction by use of concurrent GAPDH message amplification, with GAPDH probe and primers used as an internal standard.

Protein analysis. Cytosolic extracts of ventricular tissue were separated by SDS-PAGE and proteins were electrophoretically transferred to nitrocellulose filters (26). Filters were blocked in Tris-buffered saline containing $1 \%$ Tween 20 and $2 \%$ nonfat dried milk. Filters were washed and incubated with primary antibody. Primary antibodies used included: rabbit polyclonal anti-FAK antibody, murine monoclonal anti-Grb2 antibody, murine monoclonal anti-ERK antibody, rabbit polyclonal anti-p38 $\alpha$ MAPK antibody, goat polyclonal 
anti-p38 $\beta$ MAPK antibody (Santa Cruz Biotechnology Inc., Santa Cruz, California, USA), rabbit polyclonal anti-phospho p38 MAPK antibody, rabbit polyclonal anti-phospho-JNK antibody, rabbit polyclonal anti-p38 MAPK antibody, and rabbit polyclonal antiJNK antibody (Cell Signaling Technology, Beverly, Massachusetts, USA). Filters were extensively washed in Tris-buffered saline containing 1\% Tween 20 and then incubated with horseradish peroxidase-conjugated anti-rabbit, anti-goat, or anti-mouse secondary antibody (Amersham Pharmacia Biotech Inc., Piscataway, New Jersey, USA). Bands were visualized by use of the ECL system (Amersham Pharmacia Biotech Inc.) (26).

In vitro protein kinase assays. In vitro ERK activity assays were performed using a kit from Cell Signaling Technology in accordance with the manufacturer's instructions. In brief, anti-phospho-ERK immunoprecipitates were derived from ventricular cytosolic lysates obtained 7 days after TAC. $2 \mu \mathrm{g}$ of the specific substrate protein Elk-1, $200 \mu \mathrm{M}$ of ATP, and $50 \mu \mathrm{l}$ of $1 \mathrm{X}$ kinase reaction buffer were added to the immunoprecipitates. Kinase reactions were terminated after a 30 -minute incubation period, and proteins were separated by SDS-PAGE and then analyzed by immunoblotting with a specific antibody against phospho-Elk-1.

Statistical analysis. All data are reported as mean \pm SEM. Statistical analysis was performed by two-tailed Student $t$ test, $\chi^{2}$ analysis, and ANOVA where applicable. Multiple group comparison was carried out by ANOVA with the Fisher post-hoc comparison. A value of $P<0.05$ was considered to be statistically significant.

\section{Results}

Cardiac pressure overload promotes Grb2 association with FAK. Previous work demonstrated that Grb2 is expressed in heart tissue and isolated cardiomyocytes. In addition, Grb2 is recruited by activated FAK in response to mechanical stress in cultured rat neonatal cardiomyocytes or in intact isolated rat hearts $(8,14)$. We investigated the ability of pressure overload to promote the association of FAK with Grb2 in murine cardiac tissue. TAC was performed on 12-week-old wild-type $\mathrm{Grb2}^{+/+}$mice, and ventricular tissue was isolated 7 days after the surgical procedure. Anti-FAK immunoprecipitates derived from ventricular protein lysates were analyzed by anti-Grb2 immunoblotting; this revealed that Grb2 formed a complex with FAK in $\mathrm{Grb2}^{+/+}$ventricular tissue after TAC, but not after a sham operation (Figure 1a).

Reduced cardiac Grb2 protein levels in haploinsufficient mice. To investigate the role of Grb2 in the cardiac hypertrophic growth program, $\mathrm{Grb2}^{+/-}$mice were analyzed. Grb2 $2^{+/-}$mice in the $129 / \mathrm{SvJ}$ strain appear normal at birth and are fertile, and have normal cardiac structure and function at 12 weeks of age (20). Echocardiographic analysis of 12 -week-old $\mathrm{Grb2}^{+/+}$and $\mathrm{Grb2} 2^{+/-} 129 / \mathrm{SvJ}$ mice revealed that Grb2 haploinsufficient mice have normal cardiac structure and intact ventricular systolic function, with a baseline LV fractional shortening of $51 \% \pm 7 \%$. There was no evidence of cardiac hypertrophy or of reduced cardiac wall thickness in $\mathrm{Grb2}^{+/-}$ mice in the absence of pressure overload.

To determine whether disruption of one allele of the Grb2 gene resulted in reduced cardiac protein content, a series of immunoblot experiments were performed with cardiac cytosolic lysates. Anti-Grb2 immunoblotting of ventricular protein lysates demonstrated an approximately $40 \%$ reduction in Grb2 protein levels in $\mathrm{Grb2}^{+/-}$mice compared with $\mathrm{Grb2}^{+/+}$mice (Figure 1b). This decrease in Grb2 protein levels is consistent with previously published

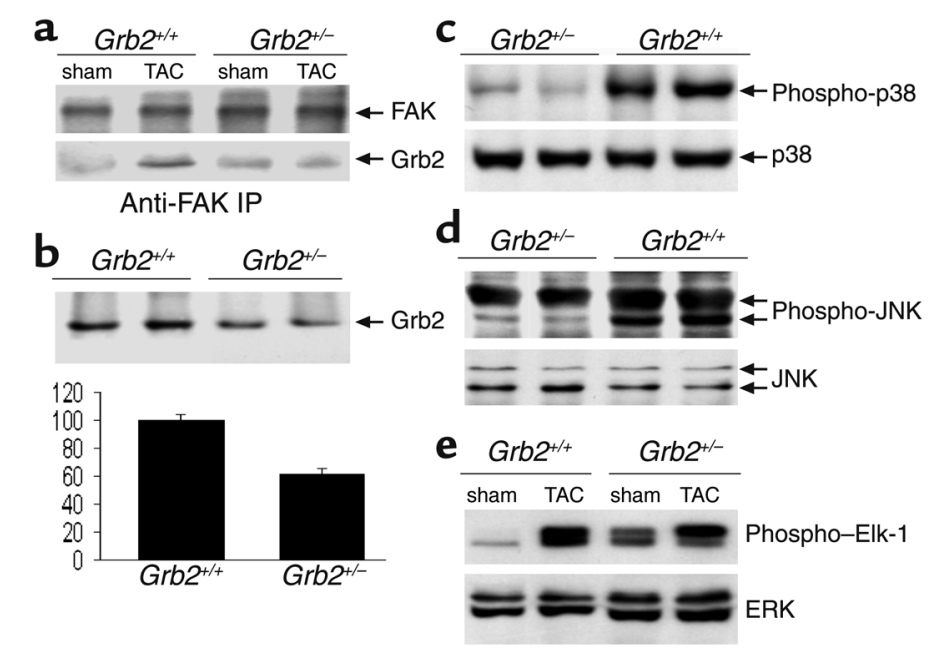

\section{Figure 1}

Biochemical characterization of Grb2 and MAPK in murine cardiac tissue 7 days after TAC or sham operation. (a) Load-induced formation of a Grb2-FAK complex. Anti-FAK immunoprecipitates (IP) derived from ventricular lysates were separated by SDS-PAGE and analyzed by immunoblotting with an anti-Grb2 antibody (lower panel). Anti-FAK immunoprecipitates were also analyzed in parallel by immunoblotting with an anti-FAK antibody (upper panel). (b) Reduced Grb2 protein content in Grb2+/- cardiac tissue. Upper panel, ventricular lysates were analyzed by immunoblotting with an anti-Grb2 antibody. Lower panel, quantification of Grb2 protein levels by densitometric analysis of immunoreactive bands. (c) Analysis of p38 MAPK activation in $\mathrm{Grb2}^{+/-}$cardiac tissue. Ventricular lysates were analyzed by immunoblotting with an anti-phospho-p38 MAPK antibody (upper panel). Lysates were also analyzed in parallel by immunoblotting with an anti-p38 MAPK (lower panel) antibody to control for protein content. (d) Analysis of JNK activation in $\mathrm{Grb2}^{+/-}$cardiac tissue. Lysates were analyzed by immunoblotting with an anti-phospho-JNK antibody (upper panel). Lysates were also analyzed in parallel by immunoblotting with an anti-JNK (lower panel) antibody to control for protein content. (e) Analysis of ERK activity in $\mathrm{Grb2}^{+/-}$cardiac tissue. Anti-ERK immunoprecipitates derived from ventricular lysates were analyzed by in vitro kinase assay by use of Elk-1 protein as a substrate. Anti-phospho-Elk-1 antibody immunoblotting was performed to assess ERK activity (upper panel). Lysates were also analyzed in parallel by immunoblotting with an anti-ERK (lower panel) antibody to control for protein content. Sham, sham operation. 

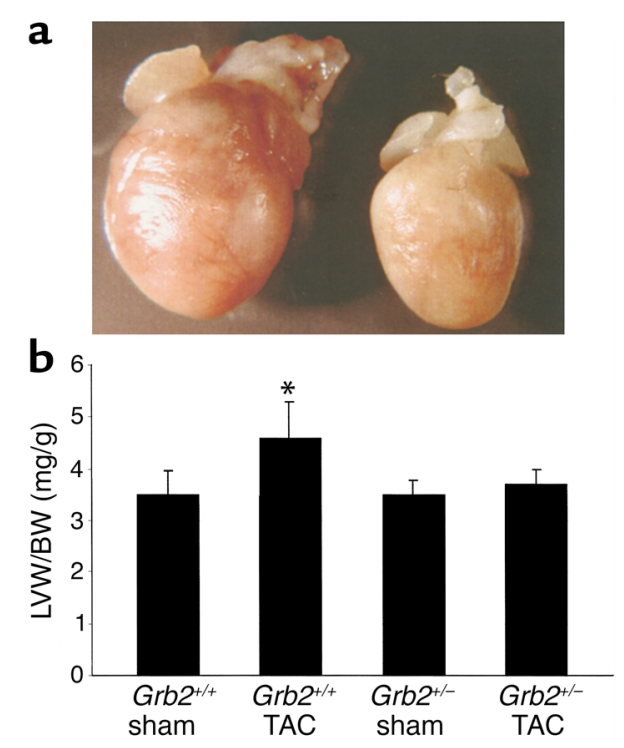

Figure 2

Reduced hypertrophic response to pressure overload in $\mathrm{Grb}^{+/-}$mice. (a) Gross morphology of hearts from $\mathrm{Grb2}^{+/+}$(left) and Grb2 $2^{+/-}$ (right) mice 7 days after TAC. (b) Morphometric analysis of cardiac hypertrophy in $\mathrm{Grb2}^{+/+}$and $\mathrm{Grb2} 2^{+/-}$mice. Seven days after TAC or sham operation, cardiac chambers were dissected and weighed to determine LWW/BW ( ${ }^{*} P<0.01$ vs. sham-operated control).

observations in $\mathrm{T}$ cells (20). In addition to reduced cardiac Grb2 protein, pressure overload-induced Grb2/FAK complex formation was markedly decreased in cardiac tissue obtained from $\mathrm{Grb2}^{+/}$ mice after TAC (Figure 1a).

Analysis of MAPK cascade activation in $\mathrm{Grb2}^{+/-}$mice after $T A C$. In wild-type mice, multiple signaling cascades are activated in response to pressure overload induced by TAC. Grb2 $2^{+/-}$mice were analyzed 7 days after TAC to determine whether there were defects in cardiac signal transduction. Grb2 $2^{+/-}$mice tolerated TAC and had a survival rate of $92 \%$ after 7 days (12 of 13 animals). Ventricular cytosolic lysates obtained from animals 7 days after TAC were analyzed by immunoblotting using antibodies against both phospho-JNK and phospho-p38 MAPK. In both cases, parallel samples were analyzed by anti-JNK and anti-p38 MAPK immunoblotting to control for protein loading. Activation of p38 MAPK and JNK was markedly decreased after TAC in $\mathrm{Grb2}^{+/-}$mice compared with $\mathrm{Grb2}^{+/+} 129 / \mathrm{SvJ}$ mice (Figure 1, $c$ and d). ERK activity in ventricular tissue samples was analyzed by in vitro kinase assay with recombinant Elk-1 protein used as a substrate. ERK activity increased in both $\mathrm{Grb2}^{+/+}$and $\mathrm{Grb2}^{+/-}$mice after TAC. In some experiments, the fold increase in ERK activity was mildly reduced in $\mathrm{Grb2}^{+/-}$ventricular tissue (Figure 1e).

Hypertrophic response of Grb2 $2^{+/}$mice to TAC. In wildtype mice, prominent LV hypertrophy develops 7 days after TAC. Typically, there is a $25-30 \%$ increase in the ratio of $\mathrm{LV}$ weight to body weight (LVW/BW) in response to pressure overload. In $\mathrm{Grb2}^{+/+} 129 / \mathrm{SvJ}$ mice, $\mathrm{LVW} / \mathrm{BW}$ increased by $31 \% 7$ days after TAC, from $3.5 \pm 0.5 \mathrm{mg} / \mathrm{g}$ to $4.6 \pm 0.7 \mathrm{mg} / \mathrm{g}$. In contrast, $\mathrm{Grb2}^{+/-}$mice were almost completely resistant to cardiac hypertrophy, and LVW/BW increased by only $5.7 \%$, from $3.5 \pm 0.3$ to $3.7 \pm 0.3 \mathrm{mg} / \mathrm{g}$ (Figure 2 , a and b). Indeed, the difference in LVW/BW between $\mathrm{Grb2}^{+/-}$ and $\mathrm{Grb2} 2^{+/+}$mice after TAC was statistically significant by Student $t$ test $(P<0.01)$. Echocardiographic analysis also demonstrated that $\mathrm{Grb2}^{+/-}$mice were resistant to TAC-induced cardiac hypertrophy. Echocardiographically determined LV mass to body weight ratio (LVM/BW) in Grb2 $2^{+/+}$mice increased by $39 \% 7$ days after TAC, but increased by only $5.4 \%$ in Grb2+/- mice (Table 1). In addition, ventricular contractility was enhanced in $\mathrm{Grb2}^{+/-}$mice 7 days after TAC with a fractional shortening of $57 \%$, compared with a fractional shortening of $48 \%$ in $\mathrm{Grb2}^{+/+}$mice (Table 1). The failure of $\mathrm{Grb2}^{+/-}$mice to exhibit cardiac hypertrophy in response to TAC was not a result of less stringent aortic constriction. Proximal systolic aortic pressures increased from $142 \pm 20 \mathrm{mmHg}$ to $196 \pm 6 \mathrm{mmHg}$ in $\mathrm{Grb2} 2^{+/-}$mice, and increased from $132 \pm 5 \mathrm{mmHg}$ to $181 \pm 12 \mathrm{mmHg}$ in Grb2 $2^{+/+}$mice.

Histological analysis of LV tissue of $\mathrm{Grb2}^{+/-}$and $\mathrm{Grb2}^{+/+}$mice was performed 7 days after TAC. Grb2 $2^{+/+}$ animals exhibited typical myocyte enlargement, myofibrillar disarray, and fibrosis after TAC, whereas $\mathrm{Grb2}^{+/-}$animals had little or no fibrosis and relatively preserved myofibrillar architecture (Figure 3).

Gene expression in $\mathrm{Grb2}^{+/-}$mice after TAC. Pressure overload leads to a variety of alterations in cardiac
Table 1

In vivo echocardiographic assessment of Grb-2+/- mice

\begin{tabular}{lcccc}
\hline & \multicolumn{2}{c}{ Sham-operated } & \multicolumn{2}{c}{ TAC } \\
& Grb-2 ${ }^{+/+}(n=6)$ & Grb-2 $^{+/-}(n=6)$ & Grb-2 $^{+/+}(n=6)$ & Grb-2 $^{+/-}(n=6)$ \\
HR (beats/min) & $628 \pm 69$ & $618 \pm 58$ & $555 \pm 87$ & $558 \pm 107$ \\
LVIDd $(\mathrm{mm})$ & $3.06 \pm 0.58$ & $3.11 \pm 0.38$ & $3.19 \pm 0.48$ & $2.70 \pm 0.49$ \\
LVIDs $(\mathrm{mm})$ & $1.59 \pm 0.56$ & $1.54 \pm 0.29$ & $1.71 \pm 0.63$ & $1.18 \pm 0.37$ \\
PWd $(\mathrm{mm})$ & $0.72 \pm 0.07$ & $0.74 \pm 0.05$ & $0.87 \pm 0.18$ & $0.83 \pm 0.07$ \\
IVSd $(\mathrm{mm})$ & $0.73 \pm 0.08$ & $0.68 \pm 0.10$ & $0.81 \pm 0.18$ & $0.79 \pm 0.18$ \\
LVM/BW $(\mathrm{mg} / \mathrm{g})$ & $2.55 \pm 0.41$ & $2.59 \pm 0.16$ & $3.54 \pm 0.92^{\mathrm{A}}$ & $2.73 \pm 0.33$ \\
FS $(\%)$ & $49.3 \pm 9.2$ & $50.5 \pm 6.28$ & $47.7 \pm 12.7$ & $57.1 \pm 8.7$ \\
LVW/BW $(\mathrm{mg} / \mathrm{g})$ & $3.5 \pm 0.5$ & $3.5 \pm 0.3$ & $4.6 \pm 0.7^{\mathrm{A}}$ & $3.7 \pm 0.3$
\end{tabular}

Echocardiographic measurements obtained from transthoracic M-mode tracings of nontransgenic Grb-2 and Grb-2 $2^{+/-}$mice 7 days after TAC or sham operation. HR indicates heart rate; LVIDd and LVIDs, end-diastolic and end-systolic LV internal dimensions, respectively; PWd and IVSd, end-diastolic posterior wall and intraventricular septal thickness; LVM, M-mode echocardiogram-derived LV mass; FS, fractional shortening; LV/BW morphometrically-determined left ventricular weight-to-body ration. ${ }^{A} P<0.05$ versus sham-operated control (Student's $t$ test) 

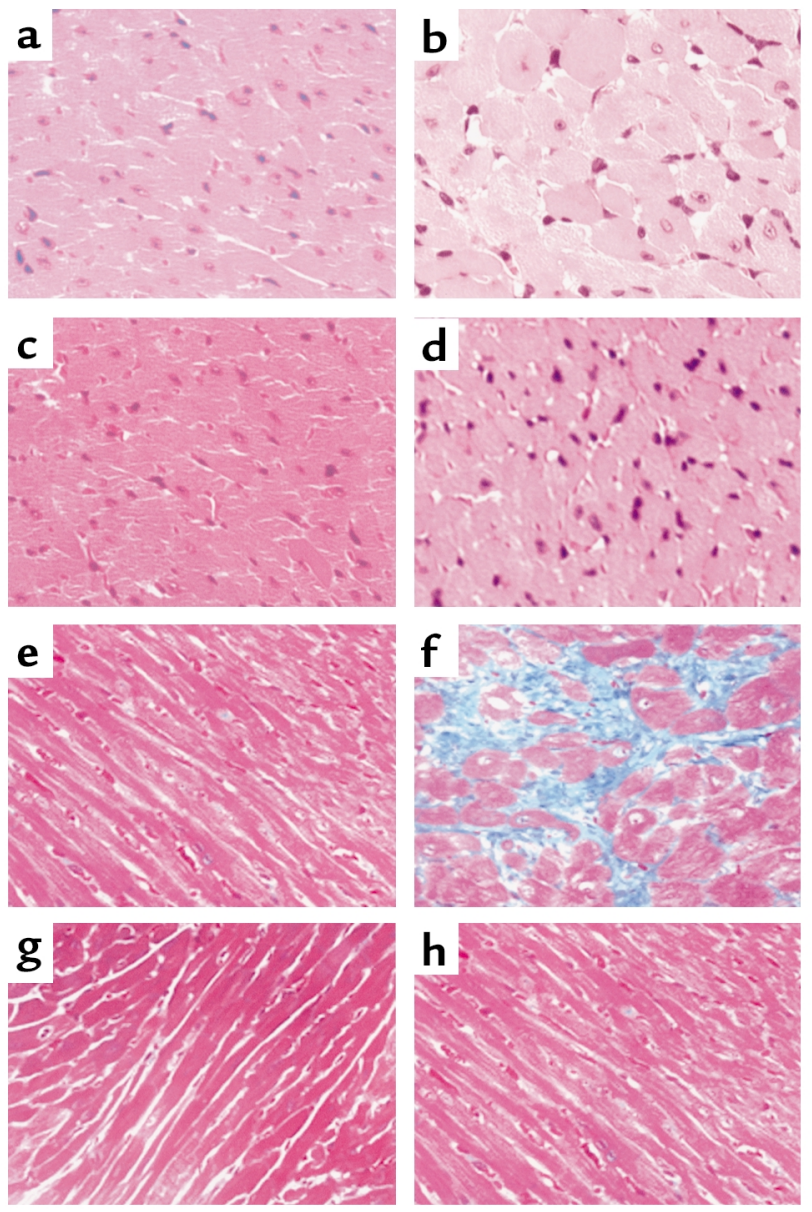

gene expression. In particular, $\beta-M H C$ and $A N F$ gene expression is typically increased in cardiac tissue in response to pressure overload. We used real-time quantitative RT-PCR to assess the expression of hypertrophic marker genes in mice after TAC. In wildtype $129 / \mathrm{SvJ}$ but not Grb2+/- mice, $\beta-M H C$ and $A N F$ gene expression was markedly induced 7 days after TAC. Indeed, $\beta-M H C$ gene expression increased by $23-$ fold and $A N F$ gene expression increased by 18 -fold in wild-type mice (Figure 4, a and b).

Transgenic mice with cardiac-specific expression of dominant negative $p 38 \alpha$ or $p 38 \beta M A P K$. Grb2 $2^{+/-}$mice are resistant to the development of cardiac hypertrophy and fibrosis after TAC and have markedly attenuated p38 MAPK and JNK signaling. Previous work demonstrated that p38 $\alpha$ MAPK is activated in cultured cardiomyocytes in

\footnotetext{
Figure 4

Inhibition of $\beta-M H C$ and $A N F$ gene expression in $\mathrm{Grb2} 2^{+/-}$cardiac tissue after pressure overload. Ventricular tissue was obtained 7 days after TAC or sham operation from $\mathrm{Grb2}^{+/+}$and $\mathrm{Grb2}^{+/-}$mice, and RNA was purified from the tissue samples. Gene expression was analyzed by use of quantitative real-time RT-PCR with specific primers and probes. GAPDH was used as an internal control in all cases. (a) $\beta-\mathrm{MHC}$ gene expression in $\mathrm{Grb2} 2^{+/+}$and $\mathrm{Grb2} 2^{+/-}$ventricular tissue. (b) ANF gene expression in $\mathrm{Grb2}^{+/+}$and $\mathrm{Grb2}^{+/-}$ventricular tissue. AU, arbitrary units.
}

\section{Figure 3}

Histological analysis of $\mathrm{Grb2}^{+/+}$and $\mathrm{Grb2} 2^{+/-} \mathrm{LV}$ tissue 7 days after TAC or sham operation. Ventricular tissue sections from (a) $\mathrm{Grb2}^{+/+}$ mouse, stained with $\mathrm{H} \& \mathrm{E}$ after sham operation; (b) $\mathrm{Grb2} 2^{+/+}$mouse, stained with H\&E after TAC; (c) Grb2 ${ }^{+/-}$mouse, stained with $\mathrm{H} \& \mathrm{E}$ after sham operation; (d) Grb2 $2^{+/-}$mouse, stained with H\&E after TAC; (e) $\mathrm{Grb2}^{+/+}$mouse, stained with Masson trichrome after sham operation; (f) $\mathrm{Grb2} 2^{+/+}$mouse, stained with trichrome after TAC. Note the increased extracellular matrix content (blue color), cardiomyocyte enlargement, and disarray. (g) Ventricular tissue section from $\mathrm{Grb2}^{+/-}$mouse, stained with trichrome after sham operation. (h) Ventricular tissue section from $\mathrm{Grb2}^{+/-}$mouse, stained with trichrome after TAC. The original magnification was $\times 400$ in all sections.

response to hypertrophic agonist stimulation $(28,29)$. In one study, p38 MAPK, but not JNK, was found to be required for phenylephrine-induced cardiomyocyte hypertrophy (28). In addition, pressure overload in rodents is known to activate cardiac p $38 \alpha$ MAPK activity (30). In contrast to work with isolated cultured cardiomyocytes, previous whole-animal work does not support the hypothesis that p38 MAPK activity promotes cardiomyocyte growth. Liao et al. recently generated transgenic mice with cardiac-specific expression of activated forms of MKK3bE or MKK6bE, kinases that act immediately upstream of $\mathrm{p} 38 \alpha$ and $\mathrm{p} 38 \beta$ MAPK (31). MKK3bE and MKK6bE transgenic mice developed cardiac fibrosis, systolic and diastolic dysfunction, and marked fetal gene induction, but no cardiac hypertrophy (31).

To test whether Grb2-facilitated p38 MAPK activation is an essential regulator of cardiac hypertrophy, we analyzed transgenic mice with cardiac-specific expression of dominant negative mutants of $\mathrm{p} 38 \alpha$ and $\mathrm{p} 38 \beta$ MAPK. In these mutants, the threonine-Xtyrosine (TXY) activation loop is altered as previously described $(21,22,26)$. The $\alpha$-MHC promoter was linked to cDNA's encoding DN-p38 $\alpha$ or DN-p38 $\beta$,

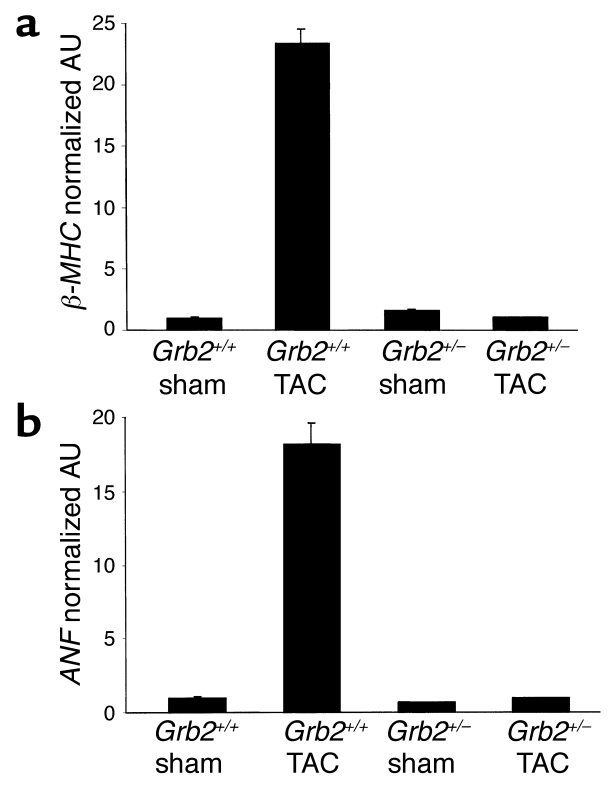




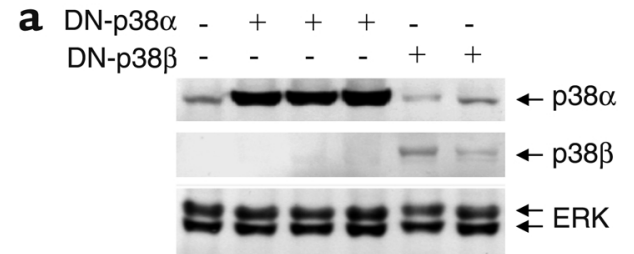

b

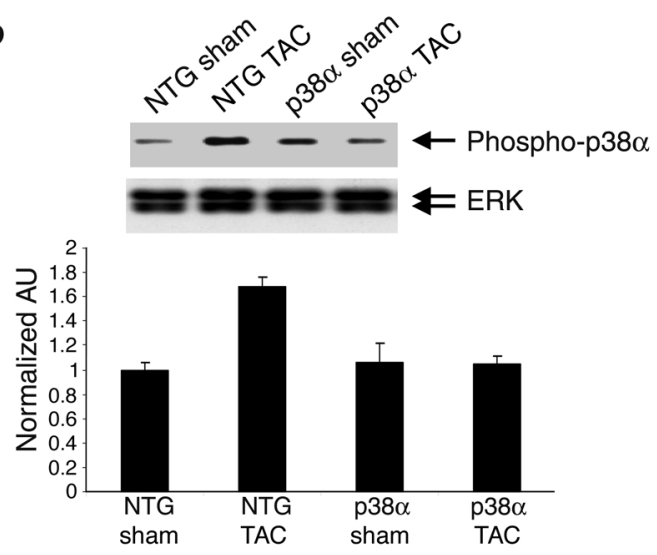

C
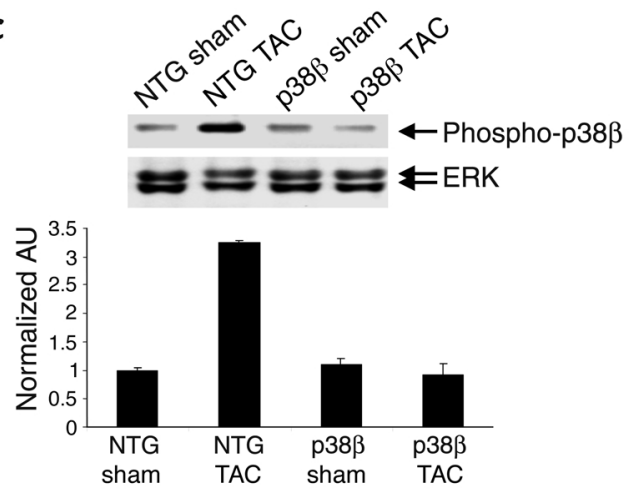

and transgenic mice in the Swiss Black strain were generated. Multiple lines were generated for each construct, and the highest-expressing lines were analyzed in this work. DN-p38 $\alpha$ mice with integration of ten copies of the transgene and DN-p38 $\beta$ mice with integration of 15 copies of the transgene were viable and fertile. Expression of the dominant negative forms of p38 was robust in transgenic mice as determined by immunoblotting of ventricular cytosolic lysates with isoform-specific anti-p38 MAPK primary antibodies (Figure 5a).

The ability of the dominant negative mutant forms of p38 MAPK to specifically inhibit native p38 $\alpha$ or p38 $\beta$ kinase activity was analyzed. Cardiac p $38 \alpha$ and p38 $\beta$ MAPK activity was analyzed by in vitro kinase assay following immunoprecipitation of phospho-p38 MAPK protein from ventricular lysates. DN-p38 $\alpha$ transgenic mice exhibited reduced p $38 \alpha$ MAPK activity and DN-p38 $\beta$ transgenic mice exhibited reduced p38 $\beta$ MAPK activity in ventricular cytosolic lysates (Figure 5, b and c).

Response of $D N-p 38 \alpha$ or $D N-p 38 \beta$ transgenic mice to TAC. In response to TAC, 15 of $19 \mathrm{DN}-\mathrm{p} 38 \alpha$ mice (79\%), and 11 of 17 DN-p38 $\beta$ mice (65\%) survived,

\section{Figure 5}

Biochemical characterization of DN-p38 $\alpha$ and DN-p38 $\beta$ transgenic mice. (a) Analysis of DN-p38 $\alpha$ and DN-p38 $\beta$ MAPK protein levels in cardiac tissue from transgenic mice. Ventricular protein lysates obtained from DN-p38 $\alpha$ mice, DN-p38 $\beta$ mice, and nontransgenic Swiss Black mice were separated by SDS-PAGE and examined by immunoblotting. Upper panel, isoform-specific anti-p38 $\alpha$ MAPK immunoblot. Middle panel, isoform-specific anti-p38 $\beta$ MAPK immunoblot. Lower panel, anti-ERK immunoblot to control for protein loading. (b) Reduced p38 $\alpha$ MAPK activity in DN-p38 $\alpha$ transgenic mice. Ventricular protein lysates were generated 7 days after TAC or sham operation in DN-p38 $\alpha$ transgenic mice or nontransgenic Swiss Black mice (NTG). Upper panel, antiphospho-p38 MAPK immunoprecipitates were analyzed by isoform-specific anti-p38 $\alpha$ MAPK immunoblotting. Middle panel, ventricular lysates were analyzed by anti-ERK immunoblotting to control for protein content. Lower panel, quantification of $\mathrm{p} 38 \alpha$ MAPK protein levels in ventricular lysates by densitometric analysis of immunoreactive bands. Data are from three experiments. (c) Reduced $p 38 \beta$ MAPK activity in DN-p38 $\beta$ transgenic mice. Upper panel, anti-phospho-p38 MAPK immunoprecipitates were analyzed by isoform-specific anti-p38 $\beta$ MAPK immunoblotting. Middle panel, ventricular lysates were analyzed by anti-ERK immunoblotting to control for protein content. Lower panel, quantification of $\mathrm{p} 38 \beta$ MAPK protein levels by densitometric analysis of immunoreactive bands. Data are from three experiments.

and these rates were similar to those observed in nontransgenic littermates. Seven days after TAC, hearts were isolated and LVW/BW was calculated as a measure of cardiac hypertrophy. As expected, LVW/BW increased by $34 \%$ in nontransgenic Swiss Black mice, from $3.5 \pm 0.2$ to $4.7 \pm 0.1 \mathrm{mg} / \mathrm{g}(P<0.01)$. DN-p38 $\alpha$ transgenic mice also developed cardiac hypertrophy after TAC (Figure 6). LVW/BW increased by $32 \%$ in DN-p38 $\alpha$ mice, from $3.4 \pm 0.1$ to $4.5 \pm 0.6 \mathrm{mg} / \mathrm{g}$ $(P<0.05)$. DN-p38 $\beta$ mice developed cardiac hypertrophy 7 days after TAC and LVW/BW increased by $51 \%$, from $3.3 \pm 0.5$ to $5.0 \pm 0.8 \mathrm{mg} / \mathrm{g}(P<0.0001)$ (Figure 6). Echocardiographic analysis confirmed that DN-p38 $\alpha$ and DN-p38 $\beta$ mice developed cardiac hypertrophy in response to pressure overload. Echocardiographically derived LVM/BW increased by $48 \%$ in nontransgenic

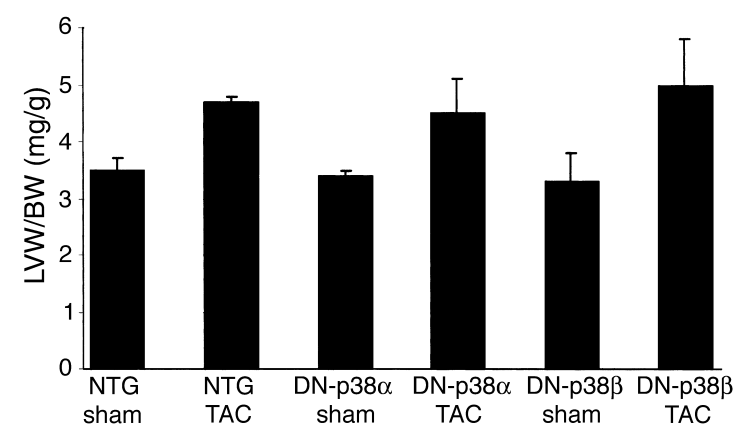

\section{Figure 6}

Preserved hypertrophic response to pressure overload in DN-p38a and DN-p38 $\beta$ transgenic mice. Morphometric analysis of cardiac hypertrophy in DN-p38 $\alpha$ mice, DN-p38 $\beta$ mice, and nontransgenic Swiss Black mice. Seven days after TAC or sham operation, cardiac chambers were dissected and weighed to determine LWW/BW. 
Table 2

In vivo echocardiographic assessment of DN-p38 mice

\begin{tabular}{lcccccc}
\hline & & & & TAC \\
& NTG $(n=8)$ & Sham-operated & & \\
DN-p38 $\alpha(n=8)$ & DN-p38 $\beta(n=8)$ & NTG $(n=8)$ & DN-p38 $\alpha(n=8)$ & DN-p38 $\beta(n=8)$ \\
HR (beats/min) & $688 \pm 36$ & $705 \pm 43$ & $673 \pm 64$ & $658 \pm 47$ & $658 \pm 37$ & $627 \pm 81$ \\
LVIDd (mm) & $3.27 \pm 0.19$ & $3.18 \pm 0.10$ & $3.18 \pm 0.30$ & $2.84 \pm 0.39$ & $3.0 \pm 0.08^{\mathrm{A}}$ & $3.05 \pm 0.28$ \\
LVIDs (mm) & $1.21 \pm 0.16$ & $1.36 \pm 0.48$ & $1.62 \pm 0.29$ & $1.25 \pm 0.35$ & $1.24 \pm 0.16$ & $1.53 \pm 0.35$ \\
PWd (mm) & $0.63 \pm 0.09$ & $0.70 \pm 0.08$ & $0.77 \pm 0.09$ & $0.80 \pm 0.13^{\mathrm{A}}$ & $0.90 \pm 0.07^{\mathrm{A}}$ & $0.94 \pm 0.07^{\mathrm{A}}$ \\
IVSd (mm) & $0.64 \pm 0.06$ & $0.69 \pm 0.1$ & $0.71 \pm 0.09$ & $0.75 \pm 0.25$ & $0.87 \pm 0.08^{\mathrm{A}}$ & $0.90 \pm 0.07^{\mathrm{A}}$ \\
LVM/BW (mg/g) & $2.28 \pm 0.31$ & $2.47 \pm 0.43$ & $2.51 \pm 0.38$ & $3.37 \pm 0.30^{\mathrm{A}}$ & $3.44 \pm 0.47^{\mathrm{A}}$ & $4.04 \pm 0.77^{\mathrm{A}}$ \\
FS (\%) & $63.2 \pm 2.7$ & $57.3 \pm 8.6$ & $49.2 \pm 5.28$ & $56.3 \pm 9.4$ & $58.6 \pm 2.2$ & $50.0 \pm 8.7$ \\
LVW/BW $(\mathrm{mg} / \mathrm{g})$ & $3.5 \pm 0.2$ & $3.4 \pm 0.1$ & $3.3 \pm 0.5$ & $4.7 \pm 0.1^{\mathrm{A}}$ & $4.5 \pm 0.5^{\mathrm{A}}$ & $5.0 \pm 0.8^{\mathrm{A}}$
\end{tabular}

Echocardiographic measurements obtained from transthoracic M-mode tracings of nontransgenic (NTG), DN-p38 $\alpha$, and DN-p38 $\beta$ transgenic mice 7 days after TAC or sham operation. ${ }^{A} P<0.05$ versus sham-operated control (Student's $t$ test).

mice, by $39 \%$ in $\mathrm{DN}-\mathrm{p} 38 \alpha$ mice, and by $61 \%$ in $\mathrm{DN}-\mathrm{p} 38 \beta$ mice 7 days after TAC (Table 2).

The ability of DN-p38 $\alpha$ and DN-p38 $\beta$ mice to exhibit cardiac hypertrophy in response to TAC was not a result of more robust aortic constriction. Proximal systolic aortic pressures increased from $127 \pm 16$ to $157 \pm 9 \mathrm{mmHg}$ in nontransgenic mice, increased from $126 \pm 9$ to $162 \pm 9 \mathrm{mmHg}$ in DN-p38 $\alpha$ mice, and increased from $129 \pm 10$ to $169 \pm 3 \mathrm{mmHg}$ in DN-p38 $\beta$ mice after TAC.

Recent work demonstrated that cardiac-specific expression of activated forms of MKK3bE or MKK6bE, kinases that act immediately upstream of p38 MAPK, resulted in a phenotype that was characterized by cardiac interstitial fibrosis, systolic contractile dysfunction, and induction of fetal marker genes (31). Surprisingly, these transgenic mice did not develop cardiac hypertrophy. To test whether DN-p38 $\alpha$ and $\mathrm{DN}$-p $38 \beta$ mice were resistant to the development of cardiac fibrosis 7 days after TAC, cardiac tissue was examined by trichrome staining. Both DN-p38 $\alpha$ and DN-p38 $\beta$ animals exhibited typical myocyte enlargement with little or no fibrosis and relatively preserved myofibrillar architecture (Figure 7). These results contrasted with those of nontransgenic Swiss Black mice that exhibited marked fibrosis as well as myocyte enlargement after TAC. Therefore, DN-p38 $\alpha$ and DN-p38 $\beta$ transgenic mice exhibited reduced cardiac

\section{Figure 7}

Histological analysis of DN-p38 $\alpha$, DN-p38 $\beta$, and nontransgenic LV tissue 7 days after TAC or sham operation. Ventricular tissue sections from (a) nontransgenic Swiss Black mouse, stained with H\&E after sham operation; (b) nontransgenic Swiss Black mouse, stained with $\mathrm{H} \& \mathrm{E}$ after TAC; (c) DN-p38 $\alpha$ transgenic mouse, stained with $\mathrm{H} \& \mathrm{E}$ after TAC; (d) DN-p38 $\beta$ transgenic mouse, stained with H\&E after TAC; (e) nontransgenic Swiss Black mouse, stained with Masson trichrome after sham operation; (f) nontransgenic Swiss Black mouse, stained with trichrome after TAC. Note the increased extracellular matrix content (blue color), cardiomyocyte enlargement, and disarray. (g) Ventricular tissue section from DN-p38 $\alpha$ transgenic mouse, stained with trichrome after TAC. (h) Ventricular tissue section from DN-p38 $\beta$ transgenic mouse, stained with trichrome after TAC. The original magnification was $\times 400$ in all sections. fibrosis in response to pressure overload, despite the fact that they both developed cardiomyocyte hypertrophy.

Gene expression analysis revealed that DN-p38 $\alpha$ and DN-p38 $\beta$ mice were not resistant to TAC-induced alterations in cardiac gene expression. In response to TAC, cardiac ANF gene expression was robustly induced in nontransgenic Swiss Black, DN-p38 $\alpha$, and DN-p38 $\beta$ mice (Figure 8a). In addition, cardiac SERCA and $M C A D$ gene expression was reduced in nontransgenic Swiss Black mice, DN-p38 $\alpha$ mice, and DN-p38 $\beta$ mice after TAC (Figure 8, b and c).
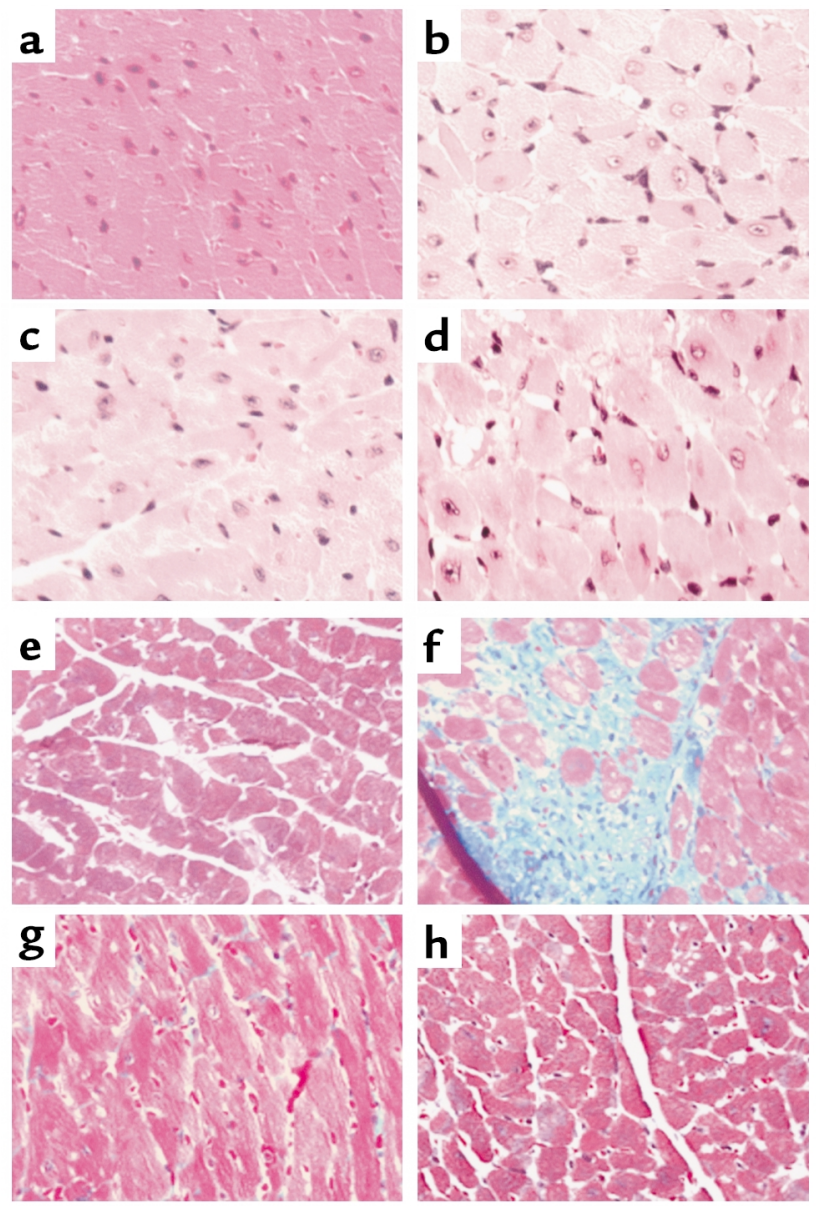
a

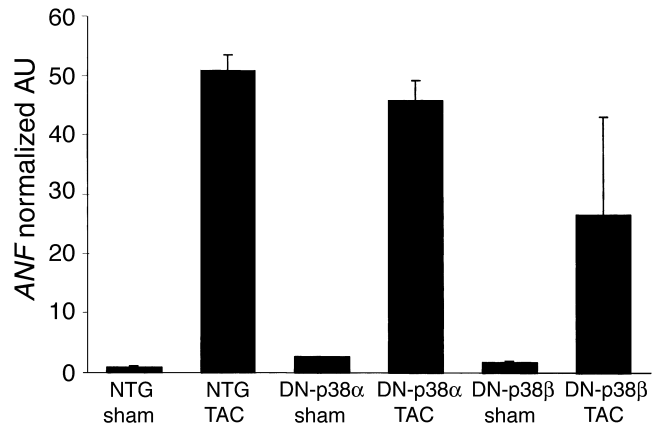

b

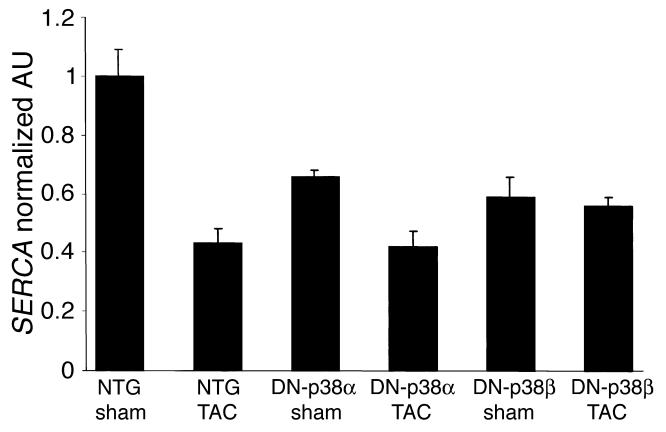

C

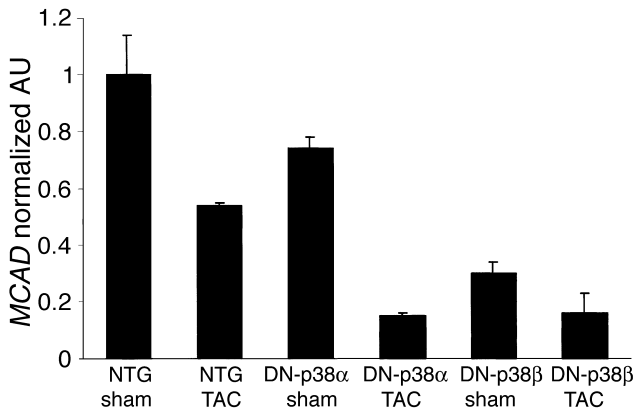

Figure 8

Pressure overload-stimulated gene expression in DN-p38 $\alpha$ and DN-p38 $\beta$ transgenic cardiac tissue and in nontransgenic Swiss Black cardiac tissue. Ventricular tissue was obtained 7 days after TAC or sham operation, and RNA was purified from the tissue samples. Gene expression was analyzed by use of quantitative real-time RT-PCR with specific primers and probes. GAPDH was used as an internal control in all cases. (a) ANF gene expression. (b) SERCA gene expression. (c) MCAD gene expression.

\section{Discussion}

Previous work suggested that cardiac pressure overload results in the activation of an integrin-mediated signaling pathway that includes the non-receptor tyrosine kinases FAK and c-Src, the scaffolding protein Grb2, the small GTPase Ras, and MAPK family members. In this work, we analyzed the roles of Grb2, p38 $\alpha$ MAPK, and p38 $\beta$ MAPK in the development of mechanical stress-induced cardiac hypertrophy by use of genetically modified mice. First, we analyzed mice that were deficient in one allele of the Grb2 gene, because mice that are deficient in both alleles die early in embryonic development.

Unlike Grb2-/- mice, Grb2 $2^{+/-}$mice survive embryonic development and are fertile $(15,20)$. In T cells and cardiac tissue, Grb2 protein levels in $\mathrm{Grb2}^{+/-}$mice are reduced by approximately $40 \%$. In vivo T cell stimulation revealed defects in lymphocyte signal transduction in $\mathrm{Grb2}^{+/-}$mice that included attenuated p38 MAPK and JNK activation, but not ERK activation (20). Similar defects in cardiac signal transduction in $\mathrm{Grb2}^{+/-}$mice were observed in this work in response to pressure overload. Indeed, we observed a marked defect in p38 MAPK and JNK activation, but intact ERK activation, in cardiac tissue 1 week after TAC. In addition, previous work with cultured Tpr-Met-transformed fibroblasts revealed that introduction of a dominant negative form of Grb2 blocked JNK but not ERK activation (32).

The defect in p38 MAPK and JNK activation observed in $\mathrm{Grb2} 2^{+/-}$mice indicates that there may be differing dose-response thresholds of various MAPK cascades to Ras activation. In this model, less Ras activity is required to activate ERK than to activate p38 or JNK. This may be due to the fact that Raf- 1 , the MAPK kinase kinase (MAPKKK) binds directly to Ras, and Raf- 1 activation may require a relatively small amount of Ras-GTP loading. In contrast, Ask 1 and other p38 and JNK MAPKKKs do not bind directly to Ras, and their activation may depend on the function of several intermediary proteins.

Cardiac hypertrophy and fibrosis did not develop in response to pressure overload in $\mathrm{Grb2}^{+/-}$mice. To further evaluate the relative role of MAPK pathways downstream of Grb2, we analyzed mice with transgenic expression of dominant negative forms of p38 MAPK in the heart. DN-p38 $\alpha$ and DN-p38 $\beta$ transgenic mice developed cardiac hypertrophy after TAC despite having markedly reduced p38 MAPK activities. Despite their ability to develop cardiac hypertrophy, DN-p38 $\alpha$ and DN-p38 $\beta$ transgenic mice did not develop cardiac fibrosis in response to pressure overload. These results demonstrate that cardiac fibrosis is not always associated with cardiac hypertrophy. These results support those of Liao et al. (31), who found that cardiac-specific expression of activated forms of MKK3bE or MKK6bE, direct upstream activators of p38 MAPK, resulted in cardiac fibrosis and systolic and diastolic dysfunction, but not cardiac hypertrophy.

Taken together, these results demonstrate that cardiac hypertrophy is dependent upon a signal transduction pathway that includes Grb2 but not p38 MAPK. One question that remains is which signaling molecules downstream of Grb2 are responsible for the growth of cardiomyocytes. In addition to the Ras activator SOS, Grb2 binds to several other important signaling molecules. For example, Grb2 binds to the Grb2-associated binder 1 (Gab1) and Grb2-associated binder 2 (Gab2) proteins, c-Abl, and dynamin (33-35). Gab2 is highly expressed in heart tissue (35). Gab1 protein is known to associate with PI3K and may be involved in activation of the Akt pathway (36). One hypothesis is that Grb2 promotes cardiac hypertrophy via a Gab1-PI3K-Akt pathway. 


\section{Acknowledgments}

This work was supported by a grant from the Pharmacia/Washington University Biomedical Research Program (to A.J. Muslin). A.J. Muslin is an Established Investigator of the American Heart Association and a recipient of the Burroughs Wellcome Fund Clinical Scientist Award in Translational Research. S. Zhang is a recipient of an American Heart Association Heartland Affiliate Beginning Grant-in-Aid.

1. Sadoshima, J., and Izumo, S. 1997. The cellular and molecular response of cardiac myocytes to mechanical stress. Annu. Rev. Physiol. 59:551-571.

2. Hennersdorf, M.G., and Strauer, B.E. 2001. Arterial hypertension and cardiac arrhythmias. J. Hypertens. 19:167-177.

3. Vakili, B.A., Okin, P.M., and Devereux, R.B. 2001. Prognostic implications of left ventricular hypertrophy. Am. Heart J. 141:334-341.

4. Sadoshima, J., Xu, Y., Slayter, H.S., and Izumo, S. 1993. Autocrine release of angiotensin II mediates stretch-induced hypertrophy of cardiac myocytes in vitro. Cell. 75:977-984.

5. Rockman, H.A., Wachhorst, S.P., Mao, L., and Ross, J., Jr. 1994. ANG II receptor blockade prevents ventricular hypertrophy and ANF gene expression with pressure overload in mice. Am. J. Physiol. Heart Circ. Physiol. 266:H2468-H2475.

6. Taylor, J.M., Rovin, J.D., and Parsons, J.T. 2000. A role for focal adhesion kinase in phenylephrine-induced hypertrophy of rat ventricular myocytes. J. Biol. Chem. 275:19250-19257.

7. Pham, C.G., et al. 2000. Striated muscle-specific $\beta 1 D$-integrin and FAK are involved in cardiac myocyte hypertrophic response pathway. Am.J. Physiol. Heart Circ. Physiol. 279:H2916-H2926.

8. Aikawa, R., et al. 2002. Integrins play a critical role in mechanical stressinduced p38 MAPK activation. Hypertension. 39:233-238.

9. Damsky, C.H., and Werb, Z. 1992. Signal transduction by integrin receptors for extracellular matrix: cooperative processing of extracellular information. Curr. Opin. Cell Biol. 4:772-781.

10. Hynes, R.O. 1992. Integrins: versatility, modulation, and signaling in cell adhesion. Cell. 69:11-25.

11. Clark, E.A., and Brugge, J.S. 1995. Integrins and signal transduction pathways: the road taken. Science. 268:233-239.

12. Kuppuswamy, D., et al. 1997. Association of tyrosine-phosphorylated c-Src with the cytoskeleton of hypertrophying myocardium. J. Biol. Chem. 272:4500-4508.

13. Schlaepfer, D.D., Hanks, S., Hunter, T., and van der Geer, P. 1994. Integrin-mediated signal transduction linked to Ras pathway by GRB2 binding to focal adhesion kinase. Nature. 372:786-791.

14. Domingos, P.P., Fonseca, P.M., Nadruz, W., Jr., and Franchini, K.G. 2002 Load-induced focal adhesion kinase activation in the myocardium: role of stretch and contractile activity. Am. J. Physiol. Heart Circ. Physiol. 282:H556-H564.

15. Cheng, A.M., et al. 1998. Mammalian Grb2 regulates multiple steps in embryonic development and malignant transformation. Cell. 95:793-803.

16. Lowenstein, E.J., et al. 1992. The SH2 and SH3 domain-containing protein GRB2 links receptor tyrosine kinases to ras signaling. Cell. 70:431-442.
17. Downward, J. 1994. The GRB2/Sem-5 adaptor protein. FEBS Lett. 338:113-117.

18. Pawson, T. 1994. Tyrosine kinase signaling pathways. Princess Takamatsu Symp. 24:303-322.

19. Schlessinger, J. 1994. SH2/SH3 signaling proteins. Curr. Opin. Genet. Dev. 4:25-30.

20. Gong, Q., et al. 2001. Disruption of T cell signaling networks and development by Grb2 haploid insufficiency. Nat. Immunol. 2:29-36.

21. Rincon, $M$., et al. 1998. Interferon-gamma expression by Th1 effector $T$ cells mediated by the p38 MAP kinase signaling pathway. EMBO J. 17:2817-2829.

22. Wang, Y., et al. 1998. Cardiac muscle cell hypertrophy and apoptosis induced by distinct members of the p38 mitogen-activated protein kinase family. J. Biol. Chem. 273:2161-2168.

23. Subramaniam, A. 1991. Tissue-specific regulation of the alpha-myosin heavy chain gene promoter in transgenic mice. J. Biol. Chem. 266:24613-24620.

24. Rogers, J.H., et al. 1999. RGS4 causes increased mortality and reduced cardiac hypertrophy in response to pressure overload. J. Clin. Invest. 104:567-576

25. Rockman, H.A., et al. 1991. Segregation of atrial-specific and inducible expression of an atrial natriuretic factor transgene in an in vivo murine model of cardiac hypertrophy. Proc. Natl. Acad. Sci.U. S. A. 88:8277-8281.

26. Xing, H., Zhang, S., Weinheimer, C., Kovacs, A., and Muslin, A.J. 2000. 14-3-3 proteins inhibit apoptosis and differentially regulate MAPK cascades. EMBO J. 19:349-358.

27. Rogers, J.H., et al. 2001. RGS4 restores cardiac contractility and normalizes hypertrophic gene induction in Gaq overexpressing mice. J. Mol. Cell. Card. 33:209-218.

28. Nemoto, S., Sheng, Z., and Lin, A. 1998. Opposing effects of Jun kinase and $\mathrm{p} 38$ mitogen-activated protein kinases on cardiomyocyte hypertrophy. Mol. Cell. Biol. 18:3518-3526.

29. Yue, T.L., et al. 2000. Extracellular signal-regulated kinase plays an essential role in hypertrophic agonists, endothelin-1 and phenylephrineinduced cardiomyocyte hypertrophy. J. Biol. Chem. 275:37895-37901.

30. Esposito, G., et al. 2001. Cardiac overexpression of a $G(q)$ inhibitor blocks induction of extracellular signal-regulated kinase and c-Jun $\mathrm{NH}(2)$-terminal kinase activity in in vivo pressure overload. Circulation. 103:1453-1458

31. Liao, P., et al. 2001. The in vivo role of p38 MAP kinases in cardiac remodeling and restrictive cardiomyopathy. Proc. Natl. Acad. Sci.U. S. A. 98: $12283-12288$.

32. Rodrigues, G.A., Park, M., and Schlessinger, J. 1997. Activation of the JNK pathway is essential for transformation by the Met oncogene. EMBO J. 16:2634-2645.

33. Holgado-Madruga, M., et al. 1996. A Grb2-associated docking protein in EGF- and insulin-receptor signaling. Nature. 379:560-564.

34. Weidner, K.M., et al. 1996. Interaction between Gab1 and the c-Met receptor tyrosine kinase is responsible for epithelial morphogenesis. Nature. 384:173-176.

35. Zhao, C., Yu, D.H., Shen, R., and Feng, G.S. 1999. Gab2, a new pleckstrin homology domain-containing adapter protein, acts to uncouple signaling from ERK kinase to Elk-1. J. Biol. Chem. 274:19649-19654.

36. Holgado-Madruga, M., Moscatello, D.K., Emlet, D.R., Dieterich, R., and Wong, A.J. 1997. Grb2-associated binder-1 mediates phosphatidylinositol 3-kinase activation and the promotion of cell survival by nerve growth factor. Proc. Natl. Acad. Sci. U. S. A. 94:12419-12424. 